\section{Lung abscess secondary to xanthogranulomatous pyelonephritis}

\author{
K Pandya, J Wilcox, H Khaw, \\ D Cleveland, O P Sharma
}

\begin{abstract}
In a patient with xanthogranulomatous pyelonephritis the initial clinical and radiological picture suggested a diagnosis of lung abscess.
\end{abstract}

Xanthogranulomatous pyelonephritis is an uncommon, atypical form of renal infection, which may be invasive and may spread to adjacent organs. ${ }^{1}$ We report a case in which it invaded the lung and presented as a lung abscess.

\section{Case report}

A 27 year old black man was admitted complaining of a productive cough, right sided chest pain, dyspnoea, and fever of three days' duration. For two months he had been taking a non-steroidal anti-inflammatory agent for pain in the right flank, and three days before admission erythromycin had been prescribed. His past medical history was otherwise unremarkable.

On examination he was normotensive but had a temperature of $39^{\circ} \mathrm{C}$, a pulse rate of $130 /$ $\mathrm{min}$, and a respiratory rate of $24 / \mathrm{min}$. There were right sided bronchial breath sounds and splinting of the right chest wall. Abdominal examination showed nothing abnormal.

Alkaline phosphatase activity was raised to $387 \mathrm{U} / \mathrm{l}$, and the total serum protein concentration was $76 \mathrm{~g} / 1$ (albumin $24 \mathrm{~g} / \mathrm{l}$ ). There was severe anaemia (haemoglobin $6.8 \mathrm{~g} / \mathrm{dl}$, packed cell volume $0 \cdot 21$ ) and leucocytosis (white cell count $\left.16.2 \times 10^{y} / 1\right)$. Prothrombin time was prolonged. The results of iron studies were consistent with anaemia of chronic disease. Urine analysis showed nothing abnormal Mixed oral flora and pneumococci were cultured from the sputum. Blood and urine cultures were negative.

Chest radiography showed a raised right hemidiaphragm with a cavitary lesion in the right lower lobe (fig 1); an ultrasound scan disclosed bilaterally enlarged and calcified kidneys. An.enlarged right kidney with a dilated collecting system and staghorn calculi was shown by computed tomography (fig 1). In addition, there was a right lower lobe lung abscess continuous with the diaphragm and the right kidney.

At surgery a right renal mass and a fibrosed right lower lobe of lung were found; both organs were adhering to the diaphragm. The lung abscess was evacuated and the right kidney was resected along with the posterior third of the diaphragm.

Bacteroides ovatus was cultured from specimens from the lung and kidney obtained at surgery and preoperative bronchoscopy. Histological examination of the kidney showed xanthogranulomatous pyelonephritis with extension of the inflammatory process into peritoneal fat and skeletal muscle (fig 2).

The patient did well after operation, with almost complete resolution of radiographic and clinical abnormalities.

\section{Discussion}

Xanthogranulomatous pyelonephritis is an uncommon, atypical form of renal infection characterised by a cellular infiltrate of lipid laden mononuclear macrophages. The inflammatory process may be invasive, spreading to adjacent structures and often forming fistulous tracts. The most commonly affected organs include the gastrointestinal tract, adjacent parts of the urinary tract, and the skin. ${ }^{12}$

Clinical symptoms include flank pain, a palpable mass in the flank, fever, anorexia, weight loss, recurrent urinary tract infections, and draining sinuses. ${ }^{2-4}$ Although the xanthogranulomatous tissue may adhere to the dia-
Department of

Medicine, Los Angeles County-University of Southern California Medical Center, Los Angeles, USA

K Pandya

J Wilcox

Khaw

D Cleveland

O P Sharma

Address for reprint requests: Dr K Pandya, Los Angeles County-University of Southern California Medica Center, 1200 North State Street, Los Angeles, CA 90033, USA

Accepted 5 October 1989

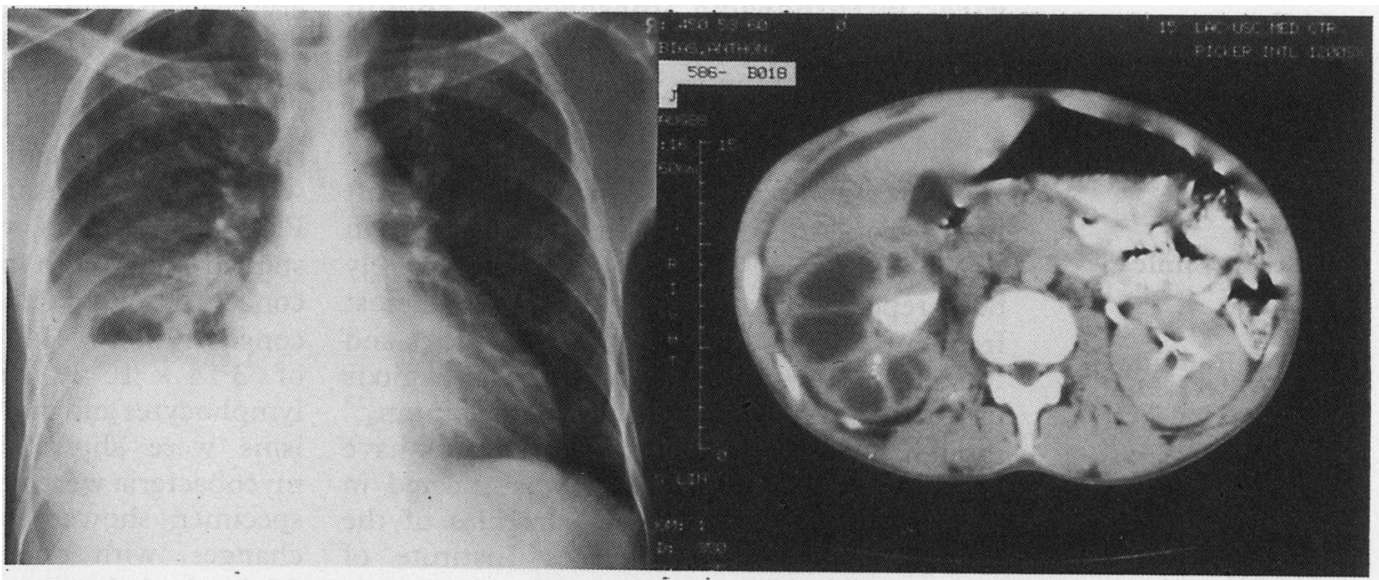

Figure 1 Left: Posteroanterior chest radiograph showing a raised right hemidiaphragm and a cavitary lesion in the right lower lobe. Right: Computed tomogram showing enlarged right kidney with a dilated collecting system and staghorn calculi. 


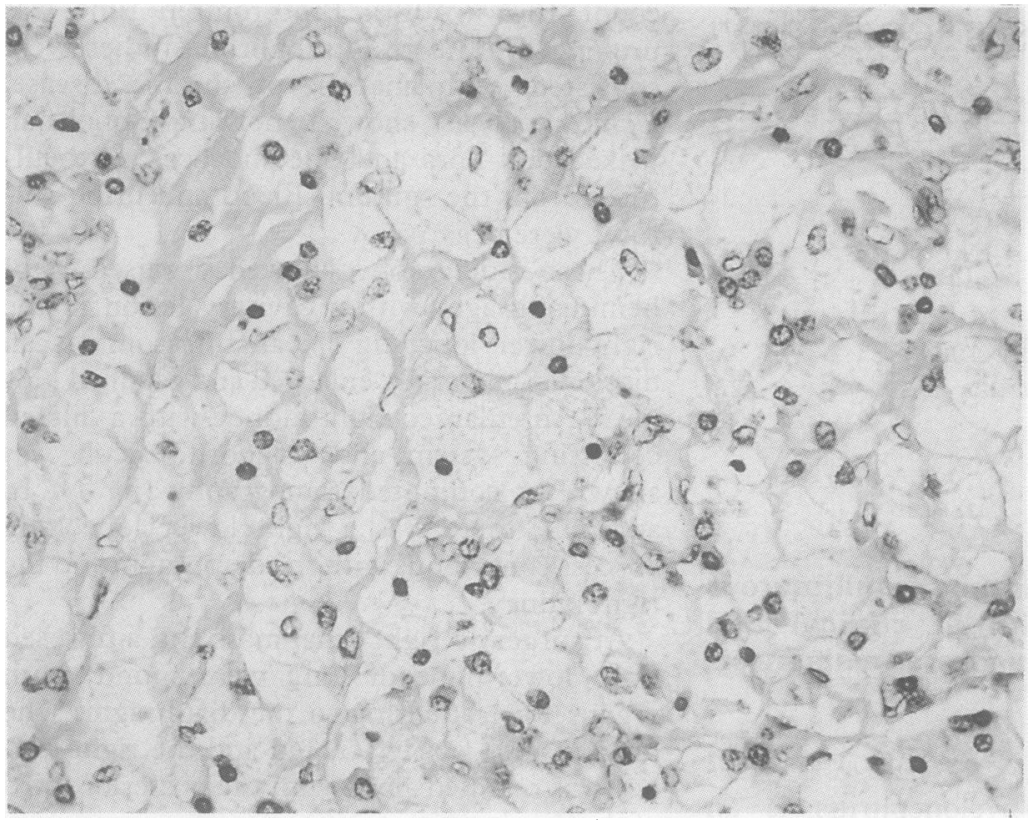

Figure 2 Microscopic section of the lesion showing the sheet like arrangement of numerous, rounded histiocytes with the abundant, foamy, clear cytoplasm and scattered plasma cells characteristic of xanthogranulomatous pyelonephritis.

phragm and has been reported, albeit rarely, to form fistulous tracts with microscopic lesions of the pleura, no case has been described where a lung abscess caused the patient's initial presentation.' Because our patient had no abnormal abdominal findings, we originally diagnosed a lung abscess on the basis of the chest radiograph. Xanthogranulomatous pyelonephritis was discovered during investigation of the raised alkaline phosphatase activity.

The aetiology of xanthogranulomatous pyelonephritis is not known but it has been produced experimentally in rats by ureteral ligation and intravenous administration of a specific Escherichia coli serotype. ${ }^{2}$ In man the most frequently encountered organisms are $E$ coli and Proteus mirabilis. ${ }^{2}$ Surgical removal of the affected kidney is the treatment of choice in most cases.

1 Parsons MA, Harris SC, Grainger RG, Ross B, Smith JAR, Williams JL. Fistula and sinus formation in Xanthogranulomatous pyelonephritis. $B r J$ Urol 1986;58:488-93.

2 Cohen MS. Granulomatous nephritis. Urol Clin North Am 1986;13:647-59.

3 Malek RS, Elder JS. Xanthogranulomatous pyelonephritis: a critical analysis of 26 cases and of the literature. $J$ Urol a critical analysis

4 Grainger RG, Longstaff AJ, Parsons MA. Xanthogranulomatous pyelonephritis: a reappraisal. Lancet 1982;i:1398-401.
Service of Clinical Pathology L Fogue

Hospital Ramón y Cajal, Universidad de Alcalá de Henares, Madrid, Spain F Ruiz-Palomo J L Calleja

Address for reprint requests: Dr F Ruiz-Palomo, Dr F Ruiz-Paic Homero 44, 2820 , Madrid, Spain.

Accepted 11 September 1989

\section{Primary liposarcoma of the lung in a young} woman

\author{
F Ruiz-Palomo, J L Calleja, L Fogue
}

\begin{abstract}
A primary liposarcoma of the lung occurred in an 18 year old girl, the first to be reported in anyone under 40 . Though rare, intrathoracic liposarcoma should be included in the differential diagnosis of pleural effusion in younger patients.
\end{abstract}

Liposarcoma is one of the more common soft tissue neoplasms of adult life, ${ }^{1}$ but it is rare in patients under 40 years of age, and has rarely been reported in anyone under $20 .^{2}$ The most frequent primary sites are the extremities and the retroperitoneum. An intrathoracic origin is unusual, and most arise in the mediastinum, ${ }^{23}$ though pleural and pulmonary tumours have been described. ${ }^{45}$ The chest was affected in only 29 of 1067 cases from the files of the United States Armed Forces Institute of Pathology. ${ }^{2}$ We describe a case of primary liposarcoma of the lung in a young woman of 18 years.

\section{Case report}

An 18 year old woman was admitted for investigation of a massive left pleural effusion. She had had a non-productive cough for three weeks and progressive dyspnoea on exertion for five days. Six days before admission she had had an episode of pyrexia $\left(38^{\circ} \mathrm{C}\right)$, which had resolved spontaneously. She denied weight loss; anorexia, drug addiction, contact with pets, allergies, articular pain, and previous tuberculosis. She had smoked 10 cigarettes a day for three years, and had had brucellosis four years previously.

On examination she appeared well, but had signs of a left sided pleural effusion. The haemoglobin concentration was $12.9 \mathrm{~g} / \mathrm{dl}$, the total white cell count $12.5 \times 10^{9} / 1$, and the erythrocyte sedimentation rate $65 \mathrm{~mm}$ in one hour. There was no biochemical abnormality. A chest radiograph showed a large left pleural effusion displacing the cardiac silhouette to the right. No malignant cells or tubercle bacilli were found on examination of the sputum. Pleural aspiration yielded a slightly blood stained fluid with a $\mathrm{pH}$ of $7 \cdot 31$, a glucose concentration of $4.2 \mathrm{mmol} / 1$, a total protein concentration of $44 \mathrm{~g} / 1$ and a white cell count of $3.14 \times 10^{9} / 1$, with a predominance of lymphocytes and mononuclear cells. No organisms were shown by Gram staining, and mycobacteria were not found. A pleural biopsy specimen showed non-specific inflammatory changes, with no evidence of malignancy. Abdominal ultrasonography showed no abnormality and bronchoscopy showed only extrinsic compression of the left bronchial tree. 